\title{
Study of magnetic domains in amorphous ribbons by scanning electron acoustic microscopy
}

\author{
M. Urchulutegui, J. Piqueras, and C. Aroca \\ Departamento de Física de Materiales, Facultad de Fisicas, Universidad Complutense, \\ 28040 Madrid, Spain
}

\begin{abstract}
(Received 10 April 1991; accepted for publication 28 May 1991)
Scanning electron acoustic microscopy (SEAM) has been used in the observation of domain walls in iron- and cobalt-based amorphous alloys ribbons. Controlled magnetic annealing experiments enabled the direct identification of domain walls in SEAM micrographs. Walls in perpendicular anisotropy regions of the high magnetostriction samples show a particular high contrast, probably related to the wall stress field.
\end{abstract}

Scanning electron acoustic microscopy (SEAM) uses a chopped electron beam to generate an acoustic signal in the specimen surface due to the interaction between the solid and the primary electron beam. ${ }^{1,2}$ This signal is usually detected by a piezoelectric transducer attached to the bottom surface of the sample and used to form a scanned image. Although in past years this technique has been applied to study many different materials, it has been only occasionally used to characterize magnetic materials as Fe-Si transformer sheets. ${ }^{3}$ In the present work, SEAM is used to investigate the domain structure in amorphous ferromagnetic ribbons with different anisotropy and magnetostrictive properties. Such observations cannot be readily performed by conventional scanning electron microscopy techniques because very high primary energies (up to 200 $\mathrm{keV}$ ) are necessary to obtain a high-contrast domain image. $^{4}$

The magnetic behavior of the amorphous alloys is determined mainly by residual stresses which might be attributed to microstructural inhomogeneities produced during rapid quenching in its preparation. ${ }^{5}$ As a consequence, a careful field annealing of the samples was in some cases necessary in order to release internal stresses and clarify considerably the magnetic domain structure of the ribbons. This allows the observation of well-separated domain walls and makes easicr the interpretation of SEAM images. Furthermore, for a better understanding of the results, SEAM micrographs were compared to those obtained by Bitter technique on the same samples.

Measurements were performed in Allied Chemical Co. amorphous ribbons with high $\left(27 \times 10^{-6}\right)$ and low $\left(0.3 \times 10^{-6}\right)$ magnetostriction constants, whose compositions are $\mathrm{Fe}_{78} \mathrm{~B}_{13} \mathrm{Si}_{9}$ and $\mathrm{CO}_{70} \mathrm{Fe}_{5} \mathrm{Ni}_{2} \mathrm{Mo}_{3} \mathrm{~B}_{5} \mathrm{Si}_{15}$, respectively. The width of the samples ranges between 4 and 8 $\mathrm{mm}$ and the thickness is about $45 \mu \mathrm{m}$. Samples were observed as-quenched and after different magnetic annealings in order to release internal stresses and to induce easy axis in different directions. The samples were annealed at $210^{\circ} \mathrm{C}$ for $8 \mathrm{~min}$ and slowly cooled for $15 \mathrm{~min}$.

SEAM observations were performed in a Cambridge S4-10 scanning electron microscope. The experimental arrangement used in this work has been described previously. ${ }^{6} \mathrm{~A}$ chopping system consisting of a pair of condensor plates and beam blanking electronics to create a periodically modulated beam is used. A square-wave voltage with frequencies from 40 to $240 \mathrm{kHz}$ is produced by a function generator. The sound signal is detected by a piezoelectric ceramic transducer (PZT) on which the samples are clamped. The signal was detected at the reference frequency $f$ or at $2 f$ by a lock-in amplifier receiving the reference signal from the function generator. Temperature measurements using a modified cooling module have also been carried out as described in Ref. 7.

In the high magnetostriction samples, the Bitter technique reveals the existence of a magnetic structure including regions with anisotropy direction in the ribbon plane and regions with anisotropy normal to the plane. Such a structure is a consequence of microstructural inhomogeneities produced during rapid quenching in the preparation

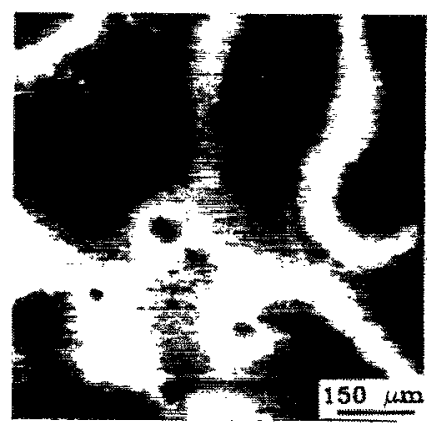

FIG. 2. SEAM image of a high magnetostrictive sample observed as-cast at $15 \mathrm{keV}$ and $157.81 \mathrm{kHz}$. 


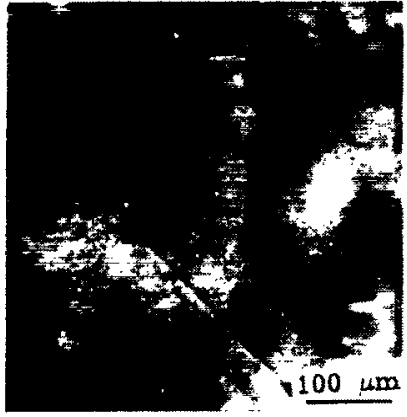

(a)

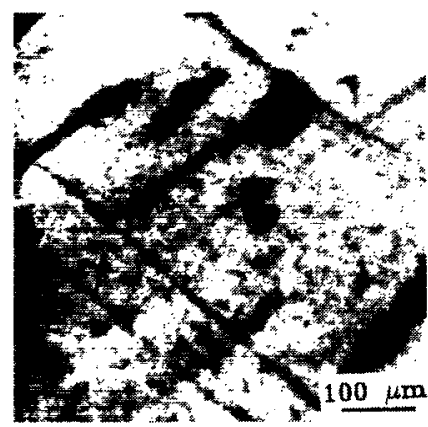

(b)

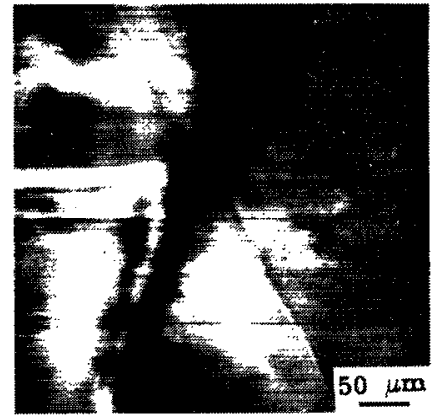

(c)
FIG. 3. High magnetostrictive sample observed after a transversal field annealing. (a) SEAM image at $15 \mathrm{keV}$ and $48.67 \mathrm{kHz}$. (b) Bitter pattern. (c) Detail of a domain wall. SEAM image at $15 \mathrm{keV}$ and $48.67 \mathrm{kHz}$. process. ${ }^{8}$ Figure 1 (a) shows a Bitter image with a region of perpendicular anisotropy embedded in a region with anisotropy in the ribbon plane. Comparison of Bitter and SEAM images allows the identification in the latter of regions with anisotropy normal to the surface, as Fig. 1(b) shows. In this figure a dark contrast with width between 10 and $20 \mu \mathrm{m}$ is observed. The dark fringes seem to be associated to domain walls or to boundaries of closure structures rather than to whole magnetic domains. Due to the SEAM capability to image stress fields through the thermoelastic signal generation mechanism, the observed contrast probably reveals the elastic stress distribution at the sides of the domain walls. The wall SEAM images have been obtained in the linear mode with the detection frequency coincident with the chopping frequency. By tuning the detector frequency to $2 f$ (nonlinear mode) a signal is obtained but the intensity is too weak to produce an image. This result is different from those reported by Balk $e t a l .^{3}$ in $\mathrm{Si}-\mathrm{Fe}$ transformer sheets. In Ref. 3 the magnetic structure has been observed in the nonlinear mode, and the black and white regions in the SEAM images were identified as domains of different directions. The observed differences indicate that in the present work the effect of elastic stresses in the domain wall dominates while in Ref. 3 the contrast can be mainly due to a nonlinear mechanism related to magnetic coupling [e.g., nonlinear variation of magnetostriction constant with temperature or the approximately quadratic dependence of strain with $M(T)$ ]. Although the SEAM images show a region distribution (Fig. 2) that seems to correspond to the zones with perpendicular and in-plane anisotropy, respectively, in the latter regions no features have been observed which could be iden-

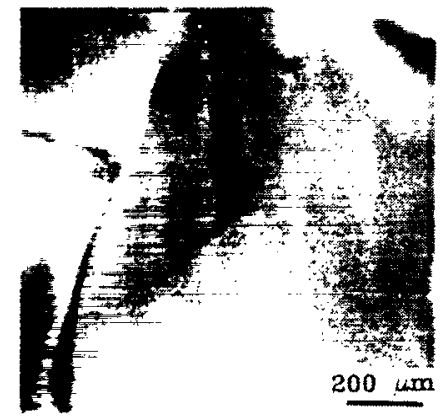

FIG. 4. Low magnetostrictive sample observed as-cast. SEAM image at $15 \mathrm{keV}$ and $23.23 \mathrm{kHz}$. tified as domain walls. In order to observe the walls in regions with anisotropy in the ribbon plane, the abovementioned annealing treatments were given with magnetic fields applied in longitudinal and transversal directions, respectively, to the rolling direction. This induces an easy axis parallel or transversal to the ribbon axis. In the annealed samples the domain walls in the regions mentioned are clearly observed in the SEAM image [Fig. 3(a)]. The Bitter image of the same sample [Fig. 3(b)] shows a similar distribution of domain walls. Figure 3 (c) shows a detail of the SEAM image of a wall whose width is about 5 $\mu \mathrm{m}$.

Figure 4 shows the SEAM image of the low magnetostriction sample without previous treatment. In addition to some high-contrast random features, probably related to mechanical stresses, a faint periodic structure (marked A) is observed which could represent a magnetic structure. As in the previous case, annealing the sample under conditions of induced anisotropy in directions longitudinal or transversal to the rolling direction produces a magnetic structure whose walls can be identified by SEAM, as shown in Fig. 5.

The present results show that magnetic structures in amorphous ribbons are readily observed by SEAM. In particular, domain walls and the distribution of their stress fields are the main features imaged in the samples used in this work. The contrast observed is probably a consequence of a thermoelastic coupling mechanism. Since the intensity of the SEAM signals has been found in several materials to be temperature dependent, ${ }^{7}$ we have measured the temperature dependence in both samples. The SEAM signal intensity in the linear mode has been found to be constant

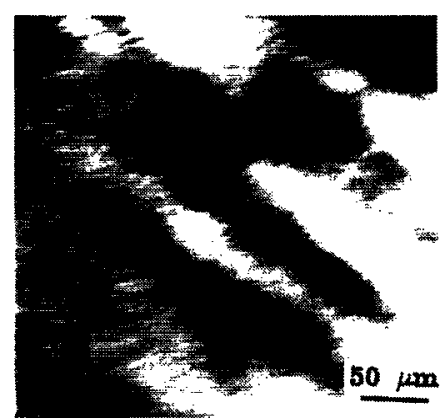

(a)

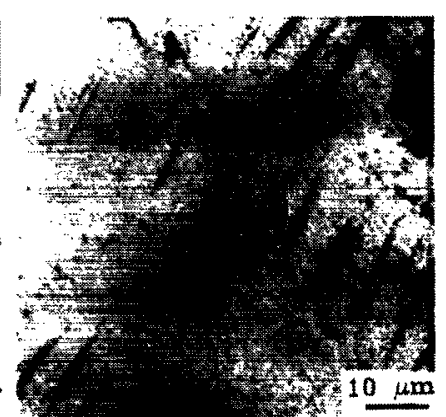

(b)
FIG. 5. Low magnetostrictive sample observed after a longitudinal field annealing. (a) SEAM image at $15 \mathrm{keV}$ and $27.57 \mathrm{kHz}$. (b) Bitter pattern. 
above $150 \mathrm{~K}$ but increases markedly below this temperature. Although this result cannot be generalized, it indicates that low-temperature SEAM measurements could be useful for the observation of magnetic structures of samples with a low SEAM signal.

This work has been supported by the Volkswagen Foundation and by CICYT (Project PB86-0151 and MAT 88-0646-CO3).

'G. S. Cargill, Nature 286, 691 (1980).

${ }^{2}$ L. J. Balk, in Aduances in Electronics and Electron Physics (Academic,
New York, 1988), Vol. 71, p. 1.

${ }^{3}$ L. J. Balk, D. G. Davies, and N. Kultscher, IEEE Trans. Magn. MAG20, 1466 (1984).

${ }^{4}$ J. D. Livingston and W. G. Morris, IEEE Trans. Magn. MAG-17, 1624 (1981).

${ }^{5}$ C. Aroca, P. S. Sánchez, and E. López, IEEE Trans. Magn. MAG-17, 1462 (1981).

${ }^{6}$ M. Urchulutegui, J. Piqueras, and J. Llopis, J. Appl. Phys. 65, 2677 (1989).

${ }^{7}$ M. Urchulutegui and J. Piqueras, J. Appl. Phys. 69, 3589 (1991)

${ }^{8}$ P. Sánchez, E. López, M. C. Sánchez-Trujillo, and C. Aroca, J. Magn. Magn. Mater. 75, 233 (1988). 